Article

\title{
NarL, a Novel Repressor for CYP108j1 Expression during PAHs Degradation in Rhodococcus sp. P14
}

\author{
Jie Kan ${ }^{1,+}$, Tao Peng ${ }^{1,+}$, Tongwang Huang ${ }^{1}$, Guangming Xiong ${ }^{2}$ and Zhong $\mathrm{Hu}^{1, *} \mathbb{C}$ \\ 1 Department of Biology, Shantou University, Shantou 515063, China; 13jkan@stu.edu.cn (J.K.); \\ tpeng@stu.edu.cn (T.P.); twhuang@stu.edu.cn (T.H.) \\ 2 Institute of Toxicology and Pharmacology for Natural Scientists, University Medical School \\ Schleswig-Holstein, 24103 Kiel, Germany; xiong-long@hotmail.com \\ * Correspondence: hzh@stu.edu.cn \\ + These authors contributed equally to this paper.
}

Received: 31 December 2019; Accepted: 30 January 2020; Published: 1 February 2020

\begin{abstract}
Rhodococcus sp. P14 was isolated from crude-oil-contaminated sediments, and a wide range of polycyclic aromatic hydrocarbons (PAHs) could be used as the sole source of carbon and energy. A key CYP450 gene, designated as cyp108j1 and involved in the degradation of PAHs, was identified and was able to hydroxylate various PAHs. However, the regulatory mechanism of the expression of cyp108j1 remains unknown. In this study, we found that the expression of cyp108j1 is negatively regulated by a LuxR (helix-turn-helix transcription factors in acyl-homoserine lactones-mediated quorum sensing) family regulator, NarL (nitrate-dependent two-component regulatory factor), which is located upstream of cyp108j1. Further analysis revealed that NarL can directly bind to the promoter region of cyp108j1. Mutational experiments demonstrated that the binding site between NarL and the cyp108j1 promoter was the palindromic sequence GAAAGTTG-CAACTTTC. Together, the finding reveal that NarL is a novel repressor for the expression of cyp108j1 during PAHs degradation.
\end{abstract}

Keywords: repressor; LuxR; PAHs; Rhodococcus; palindromic sequences

\section{Introduction}

As natural environmental products, polycyclic aromatic hydrocarbons (PAHs) have been present on the earth for many years [1]. The rapid development of industry technology and the increase in anthropogenic activities have resulted in the production of a large number of PAHs [2]. The physical and chemical properties of PAHs mean they do not degrade easily in the environment, existing ubiquitously in the air, soil, and water [3]. These PAHs can bioaccumulate through food chains, which poses a potential hazard to human health [4-7]. Bioremediation is considered a useful and available cleanup strategy and much scientific work has focused on analysis of the bacterial catabolism of PAHs [8-10]. In the last few decades, research on microbial degradation of PAHs has advanced significantly and a number of PAH-degrading isolates have been reported [1,11-13], with some of the isolates belonging to the Rhodococcus genus.

Rhodococcus can degrade many organic compounds. They are ideal candidates for enhancing the bioremediation of contaminated sites and have been proven useful for a wide range of biotransformations, such as PAHs, steroid modifications, enantioselective synthesis, and the production of amides from nitriles [14]. Water et al. used pyrene as a sole source of carbon and energy and isolated the Rhodococcus sp. UW1 from contaminated soil, which mineralized $72 \%$ of the pyrene within two weeks. At pH 7.0 and $30^{\circ} \mathrm{C}$, it showed a maximum degradation rate of $0.08 \mathrm{mg}$ pyrene $/ \mathrm{mL}$ per day. Rhodococcus sp. UW1 has a broad substrate spectrum; phenanthrene, anthracene, fluoranthene, 
and chrysene can also be used as sole sources of carbon and energy [15]. A common feature of the aerobic Rhodococcus genus is the presence of many types of monooxygenases and dioxygenases. Cytochrome p450 (CYP450) plays an important role in the process. Sylvie et al. characterized spontaneous mutants of Rhodococcus ruber unable to use ethyl tert-butyl ether (ETBE) as the sole source of carbon and energy and found that it was unable to degrade ETBE without a CYP450 gene cluster, whereas the complementation of the mutant using ethRABCD was able to degrade ETBE again, demonstrating the involvement of the Eth CYP450 system in the degradation of ETBE [16].

Regulatory proteins and regulated promoters are key elements that control the transcription of catabolic substrates, such as PAHs [17]. The regulator DfdR is one of the LuxR family proteins, which is encoded by a gene in the $d f d$ gene cluster in the dibenzofuran using Rhodococcus sp. strain YK2 and Terrabacter sp. strain YK3. The $d f d R$ gene product affects the promoter activity of the $d f d A$ gene, which is involved in the initial hydroxylation of dibenzofuran [18].

Rhodococcus sp. P14 was originally isolated from crude-oil-contaminated sediments and can use a wide range of PAHs and steroids as the sole source of carbon and energy [19-21]. Its entire genome has been sequenced [22]. Some hydroxyl products were detected during Rhodococcus sp. P14 metabolism of PAHs, suggesting that oxygenase plays an important role in the degradation process $[23,24]$. The gene cyp108j1 encoding a CYP450 was identified. Further investigation of the recombinant protein CYP108J1 proved that it is capable of the hydroxylation of a series of PAHs compounds [24].

In this study, we analyzed the promoter structure of cyp108j1 and proved that its upstream regulatory NarL has an inhibitory effect on the cyp108j1 expression. Using site-directed mutation, we identified the binding sites of NarL with the promoter cyp108j1. These findings can help us to understand more about the regulation mechanism during PAHs degradation in microorganisms.

\section{Results}

\subsection{Indentified Promoter of CYP108J1}

In previous study, we found that CYP108J1 is capable of high molecular weight PAHs oxidization and plays an important role in PAHs degradation in Rhodococcus sp. P14. In this gene cluster, two same-orientation LuxR family transcriptional regulatory genes (narl and malt) were identified upstream of cyp108j1 (Figure 1A). Using Softberry software, one promoter in the $182 \mathrm{bp}$ intergenic region in the upstream of cyp180j1 was predicted and named P3. The $-35 \mathrm{bp}$ region, $-10 \mathrm{bp}$ region, and transcriptional start site (TSS) were also predicted (the predicted transcription site A was set to 0 ) (Figure 1B).

A

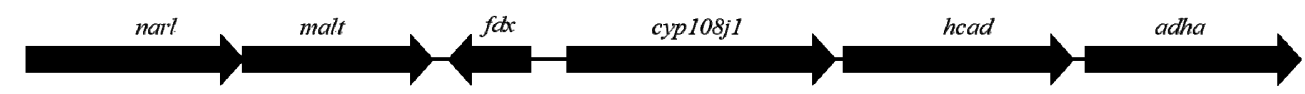

B TCGGCGTACGCATCACCACATCGACCGATTACCCCCCTCCCEGAGGGTGAAAGTTGCG $-144$

$-103$

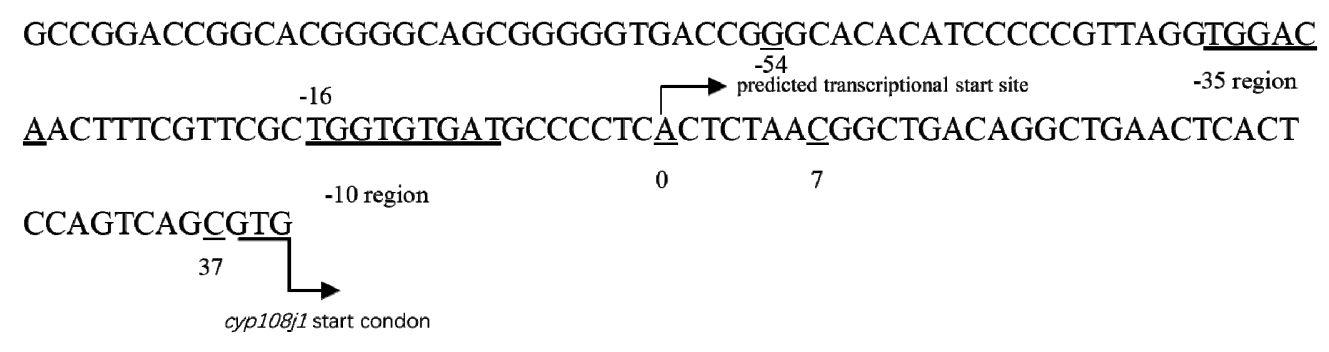

Figure 1. (A) The genomic organization of gene cluster containing cyp108j1. (B) The promoter P3 of cyp108j1. 
To detect whether promoter P3 was functional, the DNA sequence including the P3 promoter (182 bp from -144 to $37 \mathrm{bp}$ as shown in Figure 1B) was fused with egfp in the plasmid pNV18 reverse, resulting in plasmid pNV18-P3Egfp (egfp can only be transcribed from the P3 promoter), which was transferred into Eschericia coli. The E. coli strain with pNV18-P3Egfp showed the fluorescence intensity, indicating that the P3 promoter was functional (Figure 2A). The E. coli strain with pNV18-Egfp-reverse was used as the negative control (egfp was inserted into the opposite direction of the lac promoter; therefore, it could not be transcribed) and pNV18-Egfp was used as the positive control (egfp can be transcribed from the lac promoter in plasmid) (Figure 2B).
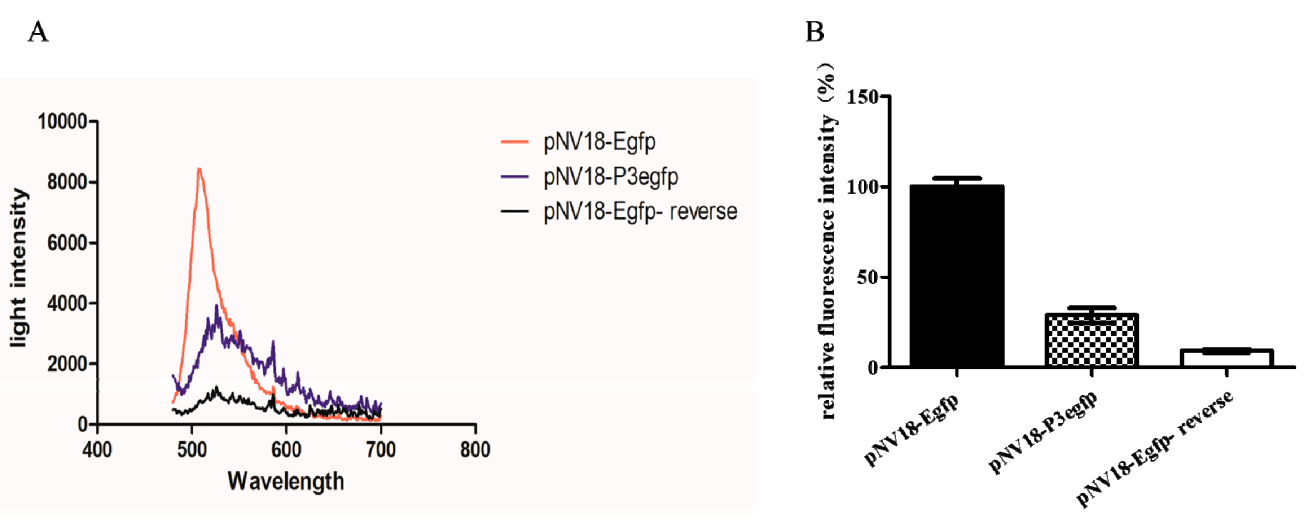

Figure 2. Detection of the function of P3 promoter. (A) The fluorescence detection of these Escherichia coli with pNV18-P3Egfp by a fluorescence spectrometer from 480 to $700 \mathrm{~nm}$. (B) The fluorescence detection of the E. coli strains with pNV18-P3Egfp, pNV18-Egfp, and pNV18-Egfp-reverse at $510 \mathrm{~nm}$. The fluorescence intensity of pNV18-Egfp was set to $100 \%$. The error bars indicate the standard deviation.

For localization of promoter P3, various deleted fragments of the $182 \mathrm{bp}$ sequence were linked with egfp directly and incorporated into the plasmid pNV18 reverse (egfp can only be transcribed from the P3 promoter), resulting in $5^{\prime}$ set and $3^{\prime}$ set plasmids (Table S1, Figure 3). These recombinant plasmids were transformed into E. coli $\mathrm{DH} 5 \alpha$ to detect the fluorescence intensity. The E. coli with pNV18-P3Egfp was used as a control. As the deletion of 10 to $30 \mathrm{bp}$ from the $3^{\prime}$ terminus and deletion of 30 to $90 \mathrm{bp}$ from the $5^{\prime}$ terminus produced 1.02- to 2.5-fold and 1.64- to 3.49-fold higher fluorescence intensity than the control, respectively. With deletion of $120 \mathrm{bp}$ from the $5^{\prime}$ terminus and $40 \mathrm{bp}$ from the $3^{\prime}$ terminus, the fluorescence intensity decreased dramatically (Figure 3 ). These results agreed with the Softberry analysis results, which proved that the promoter was located between -54 and $+7 \mathrm{bp}$ of cyp108j1 TSS (Figure 1B). 


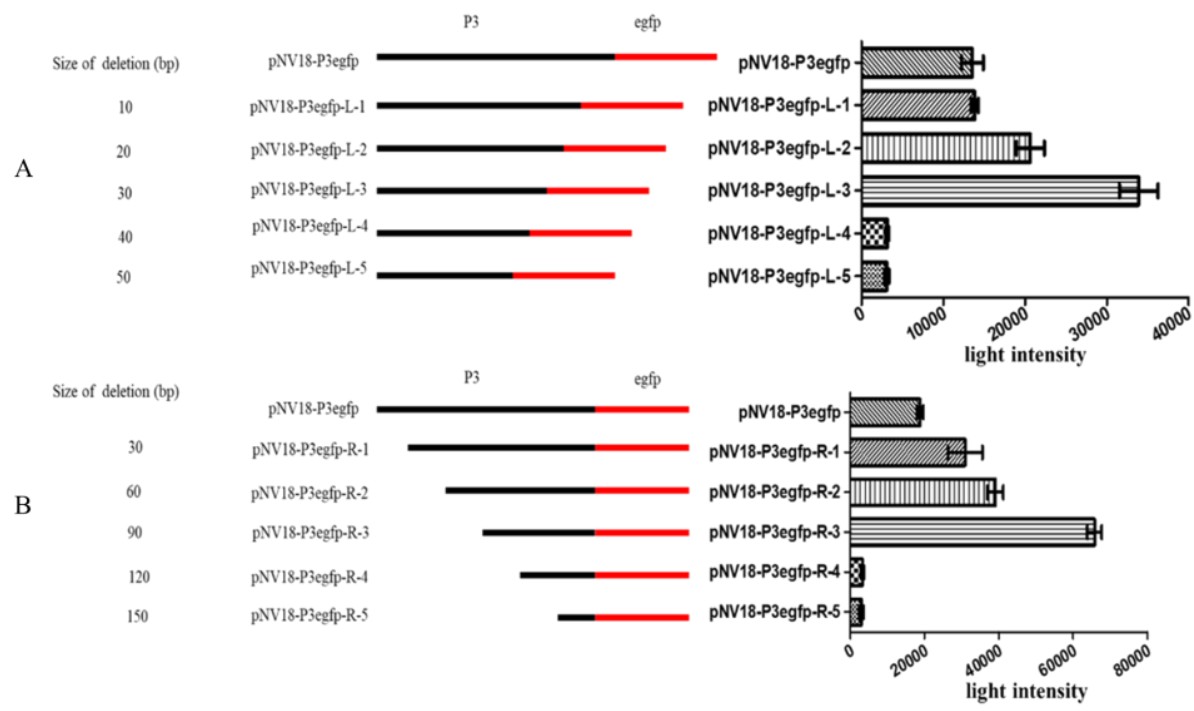

Figure 3. The activity analysis of various lengths of the P3 promoter. (A) The activity detection of the P3 promoter with deletion of 10 to $50 \mathrm{bp}$ from the $3^{\prime}$ terminus. (B) The activity detection of the P3 promoter with deletion of 30 to $150 \mathrm{bp}$ from the $5^{\prime}$ terminus. The deleted fragments of the P3 promoter were inserted into the plasmid pNV18 reverse and transferred into E. coli to detect the fluorescence. All data are presented as means \pm standard deviation (error bars).

\subsection{NarL as a Repressor for Expression of cyp108j1}

To investigate whether NarL can modulate cyp108j1 expression in Rhodococcus sp. P14, one plasmid containing the transcriptional fusion of the promoter P3 to the egfp was constructed (pACYCDuet-1-P3Egfp). The NarL protein was also transcribed from a T7 promoter in the same plasmid and the plasmid was named pACYCDuet-1-NarL-P3Egfp. The results showed that if the NarL was expressed in the cell, the fluorescence intensity decreased (Figure 4A,B), which indicates that NarL has a negative effect on the promoter activity of P3. The same experiment was performed for MalT, but no effect was observed. To further prove the influence of NarL on the expression of cyp108j1, a NarL deletion strain of Rhodococcus sp. P14 was constructed and named $\Delta$ NarL, which was confirmed by polymerase chain reaction (PCR) as shown in Figure S1. The expression levels of cyp108j1 in the wild type and $\triangle$ NarL cultured with one typical PAHs benz[a]anthracene as the only carbon source were compared to those in these strains cultured without benz[a]anthracene. The expression level of cyp108j1 increased by a 2.4 fold change compared with the wild type when it was cultured with benz[a]anthracene; this increase of cyp108j1 expression was much stronger in $\Delta$ NarL (Figure 5), which confirmed that NarL, as a repressor, has a negative effect on the expression of cyp108j1. 


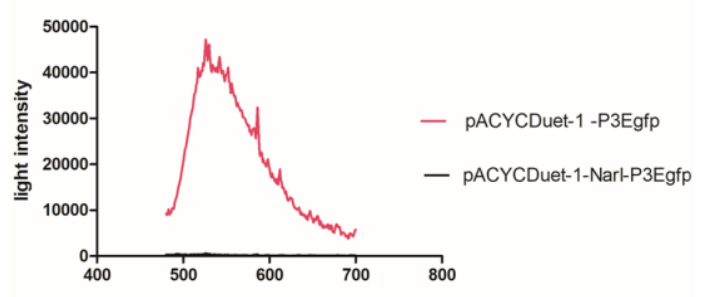

A

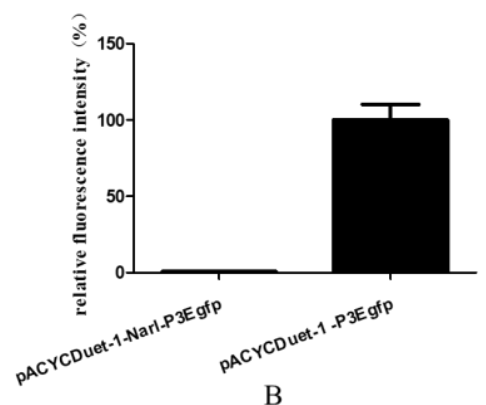

Figure 4. Analysis of the effect of NarL on P3 promoter. (A) The fluorescence detection of two E. coli strains with pACYCDuet-1-P3Egfp and pACYCDuet-1-NarL-P3Egfp using a fluorescence spectrometer from 480 to $700 \mathrm{~nm}$. (B) The fluorescence detection of two E. coli strains with pACYCDuet-1-P3Egfp and pACYCDuet-1-NarL-P3Egfp at $510 \mathrm{~nm}$. The fluorescence intensity of pACYCDuet-1-P3Egfp was set to $100 \%$. All data are presented as means \pm standard deviation (error bars).

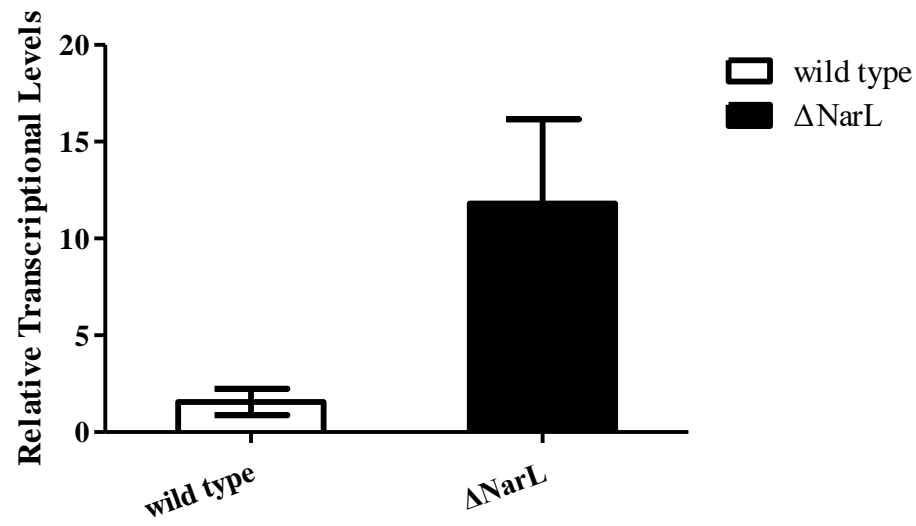

Figure 5. Detection of the mRNA levels of cyp108J1 in wild type and $\triangle$ NarL cultured with benz[a]anthracene compared to that in wild type and $\Delta$ NarL cultured without benz[a]anthracene. All data are presented as means \pm standard deviation (error bars).

\subsection{NarL Directly Binds to the Promoter of cyp108j1}

Since NarL is a regulator, the direct binding between NarL and P3 promoter was expected, and electrophoretic mobility shift assay (EMSA) was performed for confirmation. Several DNA sequences in the intergenic region were tested for their binding ability to NarL, and only one sequence, named P3-B (as shown in Figure 1B from -103 to $-16 \mathrm{pb}$ ), could produce the binding complex with NarL (data not shown). The results showed that there was no shift band when the concentration of NarL was low $(0.05$ and $0.1 \mu \mathrm{M})$; however, one shift band was observed as the concentration of NarL increased from 0.2 to $2 \mu \mathrm{M}$ (Figure 6A). The competition experiments confirmed that the binding between NarL and P3-B was a specific interaction (Figure S2).

A special palindrome sequence GAAAGTTG-CAACTTTC was identified from -96 to -88 and from -30 to -22 in the P3-B; one of these two sequences CAACTTTC covered both -10 and -35 regions (Figure 6C). As the only palindrome sequence in the $\mathrm{P3}-\mathrm{B}$, we propose that this structure might be related to the binding of NarL protein. To further test our hypothesis, a mutated DNA fragment of P3-B, named P3-B-M (Figure 6C), was designed for EMSA analysis. If the palindrome sequence GAAAGTTG was mutated to TCCCTGGT (Figure 6C), no shift band was observed (Figure 6B), which proved that the palindrome sequence was necessary for the binding between NarL and P3-B. Taking these results together, NarL, as a repressor, can specifically bind to the palindromic sequences (GAAAGTTG-CAACTTTC) upstream of cyp108j1, resulting in lower expression of cyp108j1. 


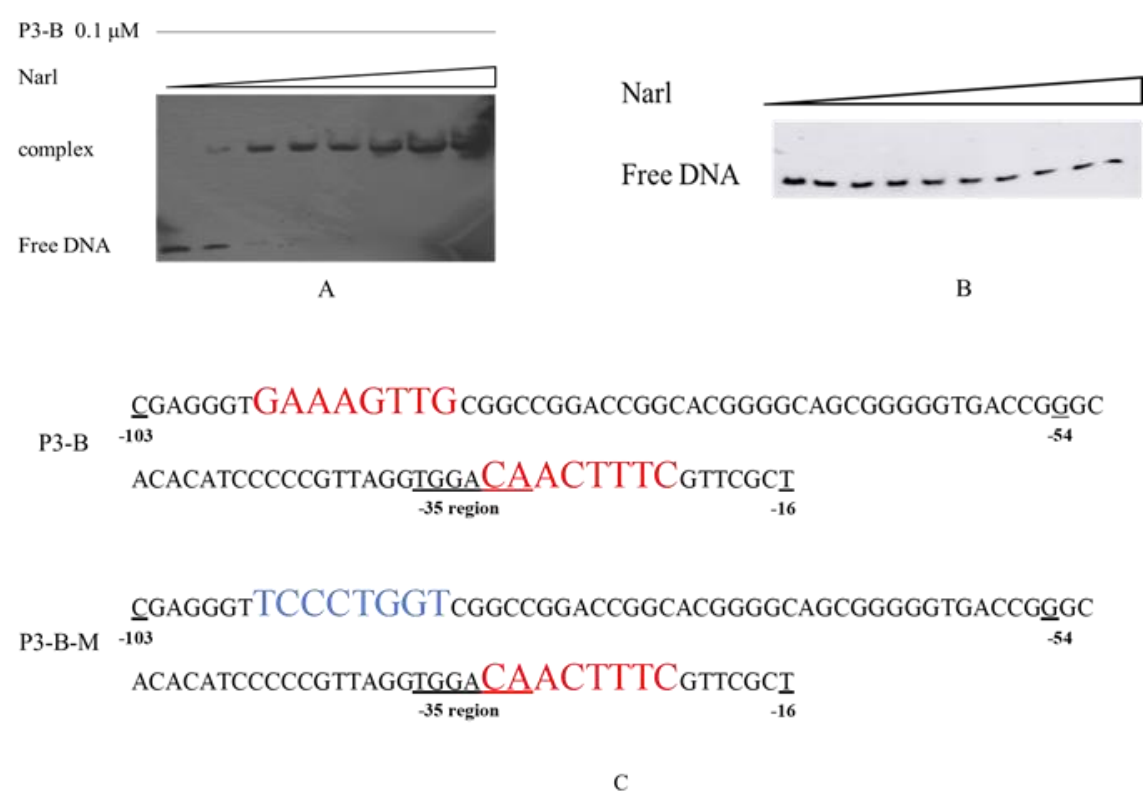

Figure 6. DNA-binding assays for DNA fragment and NarL. (A) The shift assay with NarL and P3-B. The concentration was increased from 0.05 to $2 \mu \mathrm{M}$. (B) The shift assay with NarL and P3-B-M. (C) The sequence of P3-B and its mutant P3-B-M. The red color sequences are the palindrome sequences. The blue sequence is the mutation sequence.

\section{Discussion}

According to research that can be traced back to the 1940s, a central role is played by the CYP450 monooxygenase system [25] in the degradation of a wide variety of foreign compounds such as environmental pollutants and drugs [26]. CYP450 receives the necessary electrons for oxygen cleavage and substrate hydroxylation from different redox partners [27]. Most electron transferred reactions begin with the electrons transferred from reduced form of nicotinamide-adenine dinucleotide (NADH) or nicotinamide-adenine dinucleotide phosphate (NADPH) and end with one oxygen atom from the terminal CYP450s into the substrate [28], which demonstrates that monooxygenase systems require multiple proteins to work together [29].

Some CYP450 monooxygenase in the CYP108 family has been reported to have the oxidization of PAHs activity. CYP108D1 from Novosphingobium aromaticivorans DSM12444 was reported to have the activity in the oxidation of polycyclic aromatic hydrocarbons, such as phenanthrene, biphenyl, and phenylcyclohexane [30]. CYP108A1 can effectively hydroxylate the terpene for $\alpha$-terpineol oxidation [31]. CYP108N7 from Rhodococcus NBRC 100605 is also able to catalyze the epoxidation, hydroxylation, demethylation, and dehalogenation of low molecular weight PAHs and their products [32].

In this study, we found that other genes are located in the upstream of cyp108j1 in the cluster. The promoters of these genes were also analyzed, and we found that the promoter of NarL, named P1, has a strong transcription ability in recombinant bacteria. The promoter of $f d x$ has a weak transcription ability in recombinant bacteria (data not shown). These promoters cannot bind with NarL or MalT. The SD sequences of these three promoters are not obvious, which may be related to the expression characteristics in Rhodococcus [33].

Although the cyp108j1 expression was stronger in the $\triangle$ NarL than in the wild type during PAHs degradation, we identified no significant difference in the PAHs degradation ability between $\triangle \mathrm{NarL}$ and the wild type. This may be due to the degradation of PAHs required by the synergistic action of multiple genes and a single gene up-regulation could not improve the degradation rate of Rhodococcus sp. P14.

Some other studies proved that PAHs and n-alkanes can act as small molecules which can modulate the DNA-binding and regulate genes expression [34,35]. The conformation of some regulatory proteins 
is altered by binding small molecules, which prevent them from binding to the promoters. In this study, various degradation substrates were used to attempt release the binding of NarL to the P3 promoter of cyp108j1, such as acetone, biphenyl, and hydroxy-biphenyl, but none of them succeeded (Figure S3). Phosphorylation was necessary for the function of LuxR family proteins [36,37]. Many studies have proven that phosphorylation has a strong relationship with the uptake and membrane transport of hydrocarbons [38]. We predicted that NarL, as the repressor, would be released from the promoter of cyp108j1 during PAHs degradation by an unknown mechanism; however, this still needs further study.

The CYP450 system is highly conserved in the Rhodococcus genus [39]. A TBLASTN search of the NCBI database revealed that cyp108j1 and its homologues are most found in the Rhodococcus. Among all these Rhodococcus, a conserved gene cluster contains eight genes around the CYP450, which encodes enoyl-CoA hydrase (reverse), the LuxR regulatory protein, $2 \mathrm{Fe}-2 \mathrm{~S}$ ferredoxin, CYP450, ferredoxin-NAD ${ }^{+}$reductase, alcohol dehydrogenase, and aldehyde dehydrogenase (Figure S4). The two genes encoding the LuxR family regulatory proteins (NarL and MalT) which are located in the upstream of cyp108j1 are also conserved (Figure S5A). Compared with the non-coding regions before cyp108j1 in these gene clusters, we found the palindromic sequences CAACTTTC are also conserved (Figure S5B), which implies that this regulation of NarL on cyp108j1 gene is widespread in the Rhodococcus genus.

\section{Materials and Methods}

\subsection{Chemicals and Reagents}

Benz[a]anthracene was purchased from Sigma-Aldrich (St. Louis, MO, USA). Isopropyl-d-1-thiogalactopyranoside (IPTG), T4 DNA ligase, and the restriction enzymes were purchased from TaKaRa. An RNA Extraction Kit was purchased from Promega (Promega Corporation, Madison, WI, USA). Random primers, ribonuclease inhibitor, dNTP mixture, recombinant DNase I, and SYBR ${ }^{\circledR}$ Premix Ex Taq ${ }^{\text {TM }}$ (Tli RNaseH Plus) were purchased from TransGen Biotech (TransGen Biotech, Beijing, China).

\subsection{Bacteria Strains, Plasmids, and Growth Conditions}

The bacterial strains and plasmids used in this study are shown in Table S1. The Rhodococcus sp. P14 (CGMCC NO. 2343) used in this study was isolated from crude-oil-contaminated sediments and maintained in our laboratory [19]. E. coli DH5 $\alpha$, BL21 (DE3), and their recombinants were grown in lysogeny broth (LB) medium at $37^{\circ} \mathrm{C}$. Rhodococcus sp. P14 and its NarL mutant were grown in 2216E medium [19] at $25^{\circ} \mathrm{C}$.

\subsection{Promoter Activity Analysis}

Softberry was used to identify the promoter in the upstream of cyp108j1 (http://softberry.com). The DNA fragment included the 182 bp cyp108j1 upstream region from ATG ligated to egfp and inserted into pNV18. PCR was used to generate fragments with deletions of different lengths in the $182 \mathrm{bp}$ fragment upstream of cyp108j1. Both the $5^{\prime}$ and $3^{\prime}$ deletion fragments were linked with egfp. Plasmids pACYCDuet-1 with two multiple cloning sites were used to construct the co-expression system for NarL and P3Egfp. At last, these recombinant plasmids were transformed into E. coli DH5 $\alpha$.

The generated E. coli DH5 $\alpha$ recombinants were cultured in $\mathrm{LB}$ medium for $12 \mathrm{~h}$, until an $\mathrm{OD}_{600}$ of 0.6 in $37^{\circ} \mathrm{C}$. Then, cells were harvested by centrifugation at $13,500 \times g$ for $10 \mathrm{~min}$, and the pellet was resuspended in $20 \mathrm{mM}$ Tris- $\mathrm{HCl}(\mathrm{pH} 7.85$ ) buffer, followed by disruption on ice with a sonicator for $15 \mathrm{~min}$ ( $3 \mathrm{~s}$ sonication and $3 \mathrm{~s}$ rest). At last, after centrifugation at 13,500 $\times g$ for $30 \mathrm{~min}$, supernatant was collected and determined by the Bradford method to ensure the protein concentration of each sample was the same. A fluorescence spectrometer was used to scan the green light region of the protein sample from 480 to $700 \mathrm{~nm}$, in particular at $510 \mathrm{~nm}$. Calculations and statistical analyses were performed using GraphPad software [40]. 


\subsection{Construction of NarL Mutant}

Total DNA from Rhodococcus sp. P14 cells was extracted with a kit (Dongsheng Biotech Corporation, Guangzhou, China). All primers used to construct plasmids are shown in Table S2. PCR was used to generate fragments with homologous sequences both upstream and downstream of NarL. A cassette was constructed by pNV18 promoter sequences and chloramphenicol resistance gene sequences. The chloramphenicol resistance gene was amplified from pACYCDuet-1 and then inserted into plasmid pNV18 and named pNV18-cassette (Table S1). Then, a new cassette sequence, including the promoter of plasmid pNV18-cassette to the end of chloramphenicol resistance gene, was amplified by PCR and sent to BGI (BGI Biotech Corporation, Shenzhen, China) for sequencing. At last, both homologous sequences and cassette sequences were fused together and inserted into pK18mobsacb as the suicide plasmid, named pK18mobsacb-Narl (Table S1). Electrotransformation was used for pK18mobsacb-Narl transformed into Rhodococcus sp. P14. Chloramphenicol plates was used for the first screen. We used $2216 \mathrm{E}$ medium plates for the second screen. All the colonies grown on 2216E medium plates were detected by PCR for selecting the NarL mutant (Figure S1).

\subsection{Purification of NarL}

The plasmid pET-32a was used to express the protein NarL in E. coli. The narL gene sequence, which was amplified from the total DNA of Rhodococcus sp. P14 by PCR, was inserted into pET-32a, and then the recombinant plasmid was transformed into E. coli BL21 (DE3). The E. coli BL21 (DE3) cells with pET32a-NarL were cultured in LB medium until an $\mathrm{OD}_{600}$ of 0.6 at $37^{\circ} \mathrm{C}$. Then, $1 \mathrm{mM}$ IPTG (final concentration) was used to induce the expression of the recombinant protein. The temperature of the medium was moved to $25^{\circ} \mathrm{C}$. After $16 \mathrm{~h}$ incubation, cells were harvested by centrifugation at $13,500 \times g$ for $10 \mathrm{~min}$, and the pellet was resuspended in $20 \mathrm{mM}$ Tris- $\mathrm{HCl}$ (pH 7.85) buffer, followed by disruption on ice with a sonicator for $15 \mathrm{~min}$ ( $3 \mathrm{~s}$ sonication and $3 \mathrm{~s}$ rest). The cell debris was separated from the supernatant by centrifugation and the supernatant loaded onto a nickel-nitrilotriacetic acid (Ni-NTA) agarose column (Novagen company, Madison, WI, USA) to purify the recombinant protein NarL. All proteins were deconcentrated by a protein dialysis membrane (GE Healthcare Life Sciences China, Beijing, China). Soluble NarL was assessed with sodium dodecyl sulfate-polyacrylamide gel electrophoresis (SDS-PAGE). The concentration of purified protein was determined using the Bradford method.

\subsection{EMSA}

EMSA analysis was used for detecting protein-nucleic acid interactions [41]. All the oligonucleotides containing P3-B and P3-B-M were amplified by specific primers with biotin at the $5^{\prime}$ terminus of the top strand (BGI Biotech Corporation, Shenzhen, China). Pure DNA fragments were obtained using a gel recovery kit (Dongsheng Biotech Corporation, Guangzhou, China) for further assays. A chemiluminescent EMSA kit (Beyotime Biotech Corporation, Shanghai, China) was used to test the labeled DNA and protein binding. The mixture sample contained $1 \mu \mathrm{L}$ labeled DNA $(0.1 \mu \mathrm{M}), 2 \mu \mathrm{L}$ purified protein NarL in the concentration as needed (from 0.05 to $2 \mu \mathrm{M}$ in this study), and $2 \mu \mathrm{L}$ EMSA/gel-shift binding buffer $(5 \times)$, and lastly, the nuclease-free water was added to a $10 \mu \mathrm{L}$ total. After incubation at $25{ }^{\circ} \mathrm{C}$ for $20 \mathrm{~min}$, the mixture samples were separated on nondenaturing $5 \%$ polyacrylamide gels in $0.5 \times$ Tris borate ethylene diamine tetraacetic acid (EDTA) buffer ( $\mathrm{pH} 8.3$ ) for $2 \mathrm{~h}$ at $10 \mathrm{~V} \mathrm{~cm}^{-1}$ at $4{ }^{\circ} \mathrm{C}$, and then transferred to positively charged nylon membranes for $40 \mathrm{~min}$ at 380 $\mathrm{mA}$. All operations were performed according to the manufacturer's instructions.

\subsection{RNA Isolation and Quantitative Real-Time PCR}

Rhodococcus sp. P14 and the NarL mutant grown on mineral basal medium (MBM) were supplemented with $10 \mu \mathrm{g} \mathrm{mL}^{-1}$ Benz[a]anthracene. Total RNA was isolated by using an Eastep ${ }^{\circledR}$ Super Total RNA Extraction Kit (Promega Corporation, Madison, WI, USA) according to the manufacturer's 
instructions. qPCR was performed in a 96-well plate on a Roche Light-Cycler480 system (Roche, Diagnostics, Mannheim, Germany). The $2^{-\Delta \Delta C t}$ algorithm was used to determine the relative fold changes in transcript levels. $\operatorname{rec} A$ was used as a reference gene [42].

\section{Conclusions}

Our findings demonstrate that NarL is a novel repressor for the expression of cyp108j1 during PAHs degradation. This regulation mode may exist widely in the Rhodococcus genus. Overall, these achievements could helpful construct effective bioremediation strategies in the near future.

Supplementary Materials: Supplementary Materials are available online at http://www.mdpi.com/1422-0067/21/ 3/983/s1.

Author Contributions: All authors designed the study, collected the data. and analyzed the data. J.K. and T.P. wrote the manuscript. All authors have read and agreed to the published version of the manuscript.

Acknowledgments: This research was supported by the National Natural Science Foundation of China (31670117, 31700109).

Conflicts of Interest: We declare that we have no conflicts of interest in the authorship or publication of this contribution.

\section{References}

1. Zhao, L.; Kaiser, R.I.; Xu, B.; Ablikim, U.; Ahmed, M.; Evseev, M.M.; Bashkirov, E.K.; Azyazov, V.N.; Mebel, A.M. Low-temperature formation of polycyclic aromatic hydrocarbons in Titan's atmosphere. Nat. Astron. 2018, 2, 973-979. [CrossRef]

2. Singh, S.N.; Kumari, B.; Upadhyay, S.K.; Mishra, S.; Kumar, D. Bacterial degradation of pyrene in minimal salt medium mediated by catechol dioxygenases: Enzyme purification and molecular size determination. Bioresour. Technol. 2013, 133, 293-300. [CrossRef] [PubMed]

3. Kim, K.H.; Jahan, S.A.; Kabir, E.; Brown, R.J. A review of airborne polycyclic aromatic hydrocarbons (PAHs) and their human health effects. Environ. Int. 2013, 60, 71-80. [CrossRef] [PubMed]

4. Charis, P.M.; Cliff, P.; Jennifer, F.; Kay, F.; Olaf, H.; Wan-Mohaiza, D.; William, M.B. Effect of a complex environmental mixture from coal tar containing polycyclic aromatic hydrocarbons (PAH) on the tumor initiation, PAH-DNA binding and metabolic activation of carcinogenic PAH in mouse epidermis. Carcinogenesis 2001, 22, 1077-1086.

5. Xue, W.; Warshawsky, D. Metabolic activation of polycyclic and heterocyclic aromatic hydrocarbons and DNA damage: A review. Toxicol. Appl. Pharmacol. 2005, 206, 73-93. [CrossRef]

6. Blanka, B.; Yves, G.; Pavel, R.J.; Miroslav, D.; Radim, J.S. The effect of dibenzo[a,1]pyrene and benzo[a]pyrene on human diploid lung fibroblasts: The induction of DNA adducts, expression of p53 and p21WAF1 proteins and cell cycle distribution. Mutat. Res. /Genet. Toxicol. Environ. Mutagenes. 2000, 471,57-70.

7. Ghosal, D.; Ghosh, S.; Dutta, T.K.; Ahn, Y. Corrigendum: Current State of Knowledge in Microbial Degradation of Polycyclic Aromatic Hydrocarbons (PAHs): A Review. Front. Microbiol. 2016, 7, 1837. [CrossRef]

8. Haritash, A.K.; Kaushik, C.P. Biodegradation aspects of polycyclic aromatic hydrocarbons (PAHs): A review. J. Hazard. Mater. 2009, 169, 1-15. [CrossRef]

9. Bell, T.H.; Stefani, F.O.; Abram, K.; Champagne, J.; Yergeau, E.; Hijri, M.; St-Arnaud, M. A Diverse Soil Microbiome Degrades More Crude Oil than Specialized Bacterial Assemblages Obtained in Culture. Appl. Environ. Microbiol. 2016, 82, 5530-5541. [CrossRef]

10. Hennessee, C.T.; Li, Q.X.; Parales, R.E. Effects of Polycyclic Aromatic Hydrocarbon Mixtures on Degradation, Gene Expression, and Metabolite Production in Four Mycobacterium Species. Appl. Environ. Microbiol. 2016, 82, 3357-3369. [CrossRef]

11. Kanaly, R.A.; Bartha, R.; Watanabe, K.; Harayama, S. Rapid Mineralization of Benzo[a]pyrene by a Microbial Consortium Growing on Diesel Fuel. Appl. Environ. Microbiol. 2000, 66, 4205-4211. [CrossRef] [PubMed]

12. Liang, L.; Song, X.; Kong, J.; Shen, C.; Huang, T.; Hu, Z. Anaerobic biodegradation of high-molecular-weight polycyclic aromatic hydrocarbons by a facultative anaerobe Pseudomonas sp. JP1. Biodegradation 2014, 25, 825-833. [CrossRef] [PubMed] 
13. Kim, S.J.; Kweon, O.; Jones, R.C.; Freeman, J.P.; Edmondson, R.D.; Cerniglia, C.E. Complete and integrated pyrene degradation pathway in Mycobacterium vanbaalenii PYR-1 based on systems biology. J. Bacteriol. 2007, 189, 464-472. [CrossRef] [PubMed]

14. Larkin, M.J.; Kulakov, L.A.; Allen, C.C.R. Biodegradation and Rhodococcus-masters of catabolic versatility. Curr. Opin. Biotechnol. 2005, 16, 282-290. [CrossRef] [PubMed]

15. Walter, U.; Beyer, M.; Klein, J.; Rehm, H.J. Degradation of pyrene by Rhodococcus sp. UW1. Appl. Microbiol. Biotechnol. 1991, 34, 671-676. [CrossRef]

16. Chauvaux, S.; Chevalier, F.; Le, D.C.; Fayolle, F.; Miras, I.; Kunst, F.; Beguin, P. Cloning of a genetically unstable cytochrome P-450 gene cluster involved in degradation of the pollutant ethyl tert-butyl ether by Rhodococcus ruber. J. Bacteriol. 2001, 183, 6551-6557. [CrossRef]

17. Díaz, E.; Prieto, M.A. Bacterial promoters triggering biodegradation of aromatic pollutants. Curr. Opin. Biotechnol. 2000, 11, 467-475. [CrossRef]

18. Iida, T.; Waki, T.; Nakamura, K.; Mukouzaka, Y.; Kudo, T. The GAF-like-domain-containing transcriptional regulator DfdR is a sensor protein for dibenzofuran and several hydrophobic aromatic compounds. J. Bacteriol. 2009, 191, 123-134. [CrossRef]

19. Song, X.; Xu, Y.; Li, G.; Zhang, Y.; Huang, T.; Hu, Z. Isolation, characterization of Rhodococcus sp. P14 capable of degrading high-molecular-weight polycyclic aromatic hydrocarbons and aliphatic hydrocarbons. Mar. Pollut. Bull. 2011, 62, 2122-2128. [CrossRef]

20. Ye, X.; Wang, H.; Kan, J.; Li, J.; Huang, T.; Xiong, G.; Hu, Z. A novel 17beta-hydroxysteroid dehydrogenase in Rhodococcus sp. P14 for transforming 17beta-estradiol to estrone. Chem. Biol. Interact. 2017, 276, 105-112. [CrossRef]

21. Ye, X.; Peng, T.; Feng, J.; Yang, Q.; Pratush, A.; Xiong, G.; Huang, T.; Hu, Z. A novel dehydrogenase 17beta-HSDx from Rhodococcus sp. P14 with potential application in bioremediation of steroids contaminated environment. J. Hazard. Mater. 2019, 362, 170-177. [CrossRef] [PubMed]

22. Zhang, Y.; Qin, F.; Qiao, J.; Li, G.; Shen, C.; Huang, T.; Hu, Z. Draft genome sequence of Rhodococcus sp. strain P14, a biodegrader of high-molecular-weight polycyclic aromatic hydrocarbons. J. Bacteriol. 2012, 194, 3546. [CrossRef] [PubMed]

23. Peng, T.; Luo, A.; Kan, J.; Liang, L.; Huang, T.; Hu, Z. Identification of A Ring-Hydroxylating Dioxygenases Capable of Anthracene and Benz[a]anthracene Oxidization from Rhodococcus sp. P14. J. Mol. Microbiol. Biotechnol. 2018, 28, 183-189. [CrossRef] [PubMed]

24. Luo, A.; Wu, Y.R.; Xu, Y.; Kan, J.; Qiao, J.; Liang, L.; Huang, T.; Hu, Z. Characterization of a cytochrome P450 monooxygenase capable of high molecular weight PAHs oxidization from Rhodococcus sp. P14. Process Biochem. 2016, 51, 2127-2133. [CrossRef]

25. Gotoh, O. Substrate Recognition Sites in Cytochrome P450 Family 2 (CYPB) Proteins Inferred from Comparative Analyses of Amino Acid and Coding Nucleotide Sequences. J. Biol. Chem. 1992, 267, 83-90.

26. Nebert, D.W.; Russell, D.W. Clinical importance of the cytochromes P450. Lancet. 2002, 360, 1155-1162. [CrossRef]

27. Hannemann, F.; Bichet, A.; Ewen, K.M.; Bernhardt, R. Cytochrome P450 systems-biological variations of electron transport chains. Biochim. Biophys. Acta 2007, 1770, 330-344. [CrossRef]

28. Bernhardt, R. Cytochromes P450 as versatile biocatalysts. J. Biotechnol. 2006, 124, 128-145. [CrossRef]

29. McLean, K.J.; Sabri, M.; Marshall, K.R.; Lawson, R.J.; Lewis, D.G.; Clift, D.; Balding, P.R.; Dunford, A.J.; Warman, A.J.; McVey, J.P.; et al. Biodiversity of cytochrome P450 redox systems. Biochem. Soc. Trans. 2005, 33, 796-801. [CrossRef]

30. Bell, S.G.; Yang, W.; Yorke, J.A.; Zhou, W.; Wang, H.; Harmer, J.; Copley, R.; Zhang, A.; Zhou, R.; Bartlam, M.; et al. Structure and function of CYP108D1 from Novosphingobium aromaticivorans DSM12444: An aromatic hydrocarbon-binding P450 enzyme. Acta Crystallogr. D. Biol. Crystallogr. 2012, 68, 277-291. [CrossRef]

31. Putkaradze, N.; Litzenburger, M.; Abdulmughni, A.; Milhim, M.; Brill, E.; Hannemann, F.; Bernhardt, R. CYP109E1 is a novel versatile statin and terpene oxidase from Bacillus megaterium. Appl. Microbiol. Biotechnol. 2017, 101, 8379-8393. [CrossRef] [PubMed]

32. Guo, C.; Wu, Z.L. Construction and functional analysis of a whole-cell biocatalyst based on CYP108N7. Enzym. Microb. Technol. 2017, 106, 28-34. [CrossRef] [PubMed] 
33. Ma, J.; Campbell, A.; Karlin, S. Correlations between Shine-Dalgarno Sequences and Gene Features Such as Predicted Expression Levels and Operon Structures. J. Bacteriol. 2002, 184, 5733-5745. [CrossRef] [PubMed]

34. Liang, J.L.; Nie, Y.; Wang, M.; Xiong, G.; Wang, Y.P.; Maser, E.; Wu, X.L. Regulation of alkane degradation pathway by a TetR family repressor via an autoregulation positive feedback mechanism in a Gram-positive Dietzia bacterium. Mol. Microbiol. 2016, 99, 338-359. [CrossRef]

35. Tarantini, A.; Maître, A.; Lefèbvre, E.; Marques, M.; Rajhi, A.; Douki, T. Polycyclic aromatic hydrocarbons in binary mixtures modulate the efficiency of benzo[a]pyrene to form DNA adducts in human cells. Toxicology 2011, 279, 36-44. [CrossRef]

36. Wang, H.; Yang, Y.; Xu, J.; Kong, D.; Li, Y. iTRAQ-based comparative proteomic analysis of differentially expressed proteins in Rhodococcus sp. BAP-1 induced by fluoranthene. Ecotoxicol. Environ. Saf. 2019, 169, 282-291. [CrossRef]

37. Milton, D.L. Quorum sensing in vibrios: Complexity for diversification. Int. J. Med. Microbiol. 2006, 296, 61-71. [CrossRef]

38. Yang, Z.; Wang, X.; Xu, W.; Zhou, M.; Zhang, Y.; Ma, Y.; Wang, Q. Phosphorylation of PppA at threonine 253 controls T6SS2 expression and bacterial killing capacity in the marine pathogen Vibrio alginolyticus. Microbiol. Res. 2018, 209, 70-78. [CrossRef]

39. Seth, S.H.M.B.; Edwards, J.; Rosser, S.J.; Rathbone, D.A.; Bruce, N.C. The Explosive-Degrading Cytochrome P450 System Is Highly Conserved among Strains of Rhodococcus spp. Appl. Environ. Microbiol. 2008, 74, 4550-4552. [CrossRef]

40. Swift, M.L. GraphPad Prism, Data Analysis, and Scientific Graphing. J. Chem. Inf. Comput. Sci. 1997, 37, 411-412. [CrossRef]

41. Hellman, L.M.; Fried, M.G. Electrophoretic mobility shift assay (EMSA) for detecting protein-nucleic acid interactions. Nat. Protoc. 2007, 2, 1849-1861. [CrossRef] [PubMed]

42. Livak, K.J.; Thomas, D.S. Analysis of relative gene expression data using real-time quantitative PCR and the 2(-Delta Delta C(T)) Method. Methods 2001, 25, 402-408. [CrossRef] [PubMed]

(C) 2020 by the authors. Licensee MDPI, Basel, Switzerland. This article is an open access article distributed under the terms and conditions of the Creative Commons Attribution (CC BY) license (http://creativecommons.org/licenses/by/4.0/). 